\title{
Instability of Standing Waves for a System of Nonlinear Schrödinger Equations with Three-Wave Interaction
}

\author{
By \\ Mathieu Colin ${ }^{1}$, Thierry CoLIN $^{1}$ and Masahito OHTA ${ }^{2}$ \\ $\left({ }^{1}\right.$ Université de Bordeaux, France and ${ }^{2}$ Saitama University, Japan)
}

\begin{abstract}
We consider a three-component system of nonlinear Schrödinger equations related to the Raman amplification in a plasma. In dimension $N \leq 3$, we study the orbital instability of standing wave solution of the form $\left(0,0, e^{2 i \omega t} \varphi\right)$, where $\varphi$ is a ground state of scalar nonlinear Schrödinger equation. Using time derivative instead of space derivatives to estimate nonlinear terms, we improve an instability result in our previous paper [4], and also give a simpler proof.

Key Words and Phrases. Nonlinear Schrödinger equations, Standing waves, Orbital instability.

2000 Mathematics Subject Classification Numbers. 35Q55, 35B35.
\end{abstract}

\section{Introduction}

This paper is a sequel to our previous paper [4]. We continue the study of instability of standing wave solutions for the following system of nonlinear Schrödinger equations with three-wave interaction

$$
\left\{\begin{array}{l}
i \partial_{t} u_{1}+\Delta u_{1}+\left|u_{1}\right|^{p-1} u_{1}=-\gamma u_{3} \overline{u_{2}}, \\
i \partial_{t} u_{2}+\Delta u_{2}+\left|u_{2}\right|^{p-1} u_{2}=-\gamma u_{3} \overline{u_{1}}, \\
i \partial_{t} u_{3}+\Delta u_{3}+\left|u_{3}\right|^{p-1} u_{3}=-\gamma u_{1} u_{2}
\end{array}\right.
$$

for $(t, x) \in \boldsymbol{R} \times \boldsymbol{R}^{N}$, where $N \leq 3,1<p<1+4 / N, \gamma>0$ is a constant, and $u_{1}$, $u_{2}$ and $u_{3}$ are complex valued functions of $(t, x) \in \boldsymbol{R} \times \boldsymbol{R}^{N}$. The aim in this paper is to extend the instability result presented in [4] to the three dimensional case by using a different approach which is also valid for the cases $N=1,2$. We can notice that the method we develop in the present paper is easier to handle than that of [4].

System (1) was introduced in [4] as a simplified version of a quasilinear Zakharov type system related to the Raman amplification in a plasma studied in $[2,3]$. In [4], we studied the orbital stability and instability of a semitrivial standing wave solution $\left(0,0, e^{2 i \omega t} \varphi\right)$ of $(1)$, where $\omega>0$ is a constant, and $\varphi \in H^{1}\left(\boldsymbol{R}^{N}\right)$ is a positive radial solution of 


$$
-\Delta v+2 \omega v-|v|^{p-1} v=0, \quad x \in \boldsymbol{R}^{N} .
$$

It is well-known that $\varphi \in H^{2}\left(\boldsymbol{R}^{N}\right) \cap L^{\infty}\left(\boldsymbol{R}^{N}\right)$. This fact will be used later.

Definition. We say that the standing wave solution $\left(0,0, e^{2 i \omega t} \varphi\right)$ of $(1)$ is orbitally stable if for any $\varepsilon>0$ there exists $\delta>0$ such that if $\vec{u}_{0} \in H^{1}\left(\boldsymbol{R}^{N}, \boldsymbol{C}^{3}\right)$ and $\left\|\vec{u}_{0}-(0,0, \varphi)\right\|_{H^{1}}<\delta$, then the solution $\vec{u}(t)$ of (1) with $\vec{u}(0)=\vec{u}_{0}$ satisfies

$$
\sup _{t \geq 0} \inf _{\theta \in \boldsymbol{R}, y \in \boldsymbol{R}^{N}}\left\|\vec{u}(t)-\left(0,0, e^{i \theta} \varphi(\cdot+y)\right)\right\|_{H^{1}}<\varepsilon .
$$

Otherwise, $\left(0,0, e^{2 i \omega t} \varphi\right)$ is called orbitally unstable.

In [4], we proved the following.

Theorem 1 (Theorem 3 in [4]). Let $1 \leq N \leq 3,1<p<1+4 / N, \omega>0$, and let $\varphi$ be the positive radial solution of (2). Then, there exists a positive constant $\gamma^{*}=\gamma^{*}(N, p, \omega)$ satisfying the following.

(i) If $0<\gamma<\gamma^{*}$, then the standing wave solution $\left(0,0, e^{2 i \omega t} \varphi\right)$ of (1) is orbitally stable.

(ii) Assume further that $N \leq 2$ and $p>2$. If $\gamma>\gamma^{*}$, then the standing wave solution $\left(0,0, e^{2 i \omega t} \varphi\right)$ of (1) is orbitally unstable.

Remark that the additional assumption $N \leq 2$ and $p>2$ in Theorem 1 (ii) is related to the regularity of the nonlinearity $|u|^{p-1} u$, and it was needed to estimate the nonlinear terms of the linearized equation (4) around $\left(0,0, e^{2 i \omega t} \varphi\right)$ (see Lemmas 12 and 13 in [4]). Note that the function $z \mapsto|z|^{p-1} z$ is not smooth enough under our assumption $p<1+4 / N$. The purpose in the present paper is to remove the technical assumption $N \leq 2$ and $p>2$ from Theorem 1 (ii). In [4], the nonlinearity $F$ of Equation (4) is estimated in $H^{1}$ (resp. $H^{1+\varepsilon}$ ) in dimension 1 (resp. dimension 2). The main idea here is to use time derivative instead of space derivatives so that $F$ is estimated only in $L^{2}$ as well as its time derivative $\partial_{t} F$ (see Lemmas 5 and 8 below). This kind of idea has been used for construction of $H^{2}$ solutions to nonlinear Schrödinger equations by Kato [7] and Tsutsumi [8] (see also Section 4.8 in [1]). As a consequence, we do not need to introduce a Besov space framework to handle the following extended instability result.

Theorem 2. Let $1 \leq N \leq 3,1<p<1+4 / N, \omega>0$, and let $\varphi$ be the positive radial solution of (2). Let $\gamma^{*}=\gamma^{*}(N, p, \omega)$ be the same positive constant as in Theorem 1. If $\gamma>\gamma^{*}$, then the standing wave solution $\left(0,0, e^{2 i \omega t} \varphi\right)$ of (1) is orbitally unstable.

The rest of this paper is organized as follows. In Section 2, we first prove some basic estimates on nonlinearities, and then recall some results proved in 
[4]. In Section 3, we give the proof of Theorem 2 along the lines of Section 6 in Grillakis, Shatah and Strauss [6] together with the estimates proved in Section 2. In the sequel, $C, C_{1}, C_{2}, \ldots$ denote positive constants which depend only on $N, p, \gamma, \omega$ and $\|\varphi\|_{L^{\infty}}$.

\section{Preliminaries}

In what follows, we put $f(z)=|z|^{p-1} z$ for $z \in C$. Since $p>1$, the function $f: \boldsymbol{C} \rightarrow \boldsymbol{C}$ is of class $C^{1}$ in the real sense. For $z \in \boldsymbol{C}$, the $\boldsymbol{R}$-linear map $f^{\prime}(z): C \rightarrow C$ is given by

$$
f^{\prime}(z) w=\frac{\partial f}{\partial z}(z) w+\frac{\partial f}{\partial \bar{z}}(z) \bar{w}=\frac{p+1}{2}|z|^{p-1} w+\frac{p-1}{2}|z|^{p-3} z^{2} \bar{w}
$$

for $w \in \boldsymbol{C}$, and $f^{\prime}$ satisfies

$$
\left|f^{\prime}(z)-f^{\prime}(w)\right| \leq C \begin{cases}|z-w|^{p-1} & \text { if } 1<p \leq 2 \\ \left(|z|^{p-2}+|w|^{p-2}\right)|z-w| & \text { if } p>2\end{cases}
$$

for all $z, w \in C$ (see Lemma 2.4 in [5] for the case $p \leq 2$ ).

Lemma 3. Let $N \leq 3$. There exists a constant $C$ such that

$$
\left\|f^{\prime}(\varphi+v)-f^{\prime}(\varphi)\right\|_{L^{\infty}} \leq C\left(\|v\|_{H^{2}}+\|v\|_{H^{2}}^{p-1}\right)
$$

for all $v \in H^{2}\left(\boldsymbol{R}^{N}, \boldsymbol{C}\right)$.

Proof. By (3), we have

$$
\left\|f^{\prime}(\varphi+v)-f^{\prime}(\varphi)\right\|_{L^{\infty}} \leq C \begin{cases}\|v\|_{L^{\infty}}^{p-1} & \text { if } 1<p \leq 2, \\ \left(\|\varphi\|_{L^{\infty}}^{p-2}+\|v\|_{L^{\infty}}^{p-2}\right)\|v\|_{L^{\infty}} & \text { if } p>2 .\end{cases}
$$

Since $N \leq 3$, by the embedding $H^{2}\left(\boldsymbol{R}^{N}\right) \hookrightarrow L^{\infty}\left(\boldsymbol{R}^{N}\right)$, we have the desired estimate.

As in [4], to prove the orbital instability of $\left(0,0, e^{2 i \omega t} \varphi\right)$, we make the following change of variables

$$
\vec{u}(t)=\left(e^{i \omega t} v_{1}(t), e^{i \omega t} v_{1}(t), e^{2 i \omega t}\left(\varphi+v_{2}(t)\right)\right)
$$

in (1). Note that (1) is symmetric with respect to $u_{1}$ and $u_{2}$. Then, the equations for $\left(v_{1}, v_{2}\right)$ read

$$
\partial_{t} v_{1}=\mathscr{L}_{1} v_{1}+F_{1}\left(v_{1}, v_{2}\right), \quad \partial_{t} v_{2}=\mathscr{L}_{2} v_{2}+F_{2}\left(v_{1}, v_{2}\right)
$$


where the linear terms $\mathscr{L}_{1} v_{1}$ and $\mathscr{L}_{2} v_{2}$ are given by

$$
\begin{aligned}
& \mathscr{L}_{1} v=-i(-\Delta v+\omega v-\gamma \varphi \bar{v}), \\
& \mathscr{L}_{2} v=-i\left\{-\Delta v+2 \omega v-f^{\prime}(\varphi) v\right\},
\end{aligned}
$$

and the nonlinear terms $F_{1}\left(v_{1}, v_{2}\right)$ and $F_{2}\left(v_{1}, v_{2}\right)$ are given by

$$
\begin{aligned}
& F_{1}\left(v_{1}, v_{2}\right)=i\left(\gamma \overline{v_{1}} v_{2}+\left|v_{1}\right|^{p-1} v_{1}\right), \\
& F_{2}\left(v_{1}, v_{2}\right)=i\left\{\gamma v_{1}^{2}+f\left(\varphi+v_{2}\right)-f(\varphi)-f^{\prime}(\varphi) v_{2}\right\} .
\end{aligned}
$$

We write (4) as

$$
\begin{gathered}
\partial_{t} \mathbf{v}=\mathscr{L} \mathbf{v}+F(\mathbf{v}), \\
\mathbf{v}=\left(v_{1}, v_{2}\right), \quad \mathscr{L} \mathbf{v}=\left(\mathscr{L}_{1} v_{1}, \mathscr{L}_{2} v_{2}\right), \quad F(\mathbf{v})=\left(F_{1}(\mathbf{v}), F_{2}(\mathbf{v})\right) .
\end{gathered}
$$

We begin with basic estimates on $\mathscr{L}$ and $F$.

Lemma 4. There exists a constant $C$ such that

$$
\|\mathbf{v}\|_{H^{2}} \leq\|\mathscr{L} \mathbf{v}\|_{L^{2}}+C\|\mathbf{v}\|_{L^{2}}, \quad\|\mathscr{L} \mathbf{v}\|_{L^{2}} \leq C\|\mathbf{v}\|_{H^{2}}
$$

for all $\mathbf{v} \in H^{2}\left(\boldsymbol{R}^{N}, \boldsymbol{C}^{2}\right)$.

Proof. Since $\varphi \in H^{1}\left(\boldsymbol{R}^{N}\right)$ is a solution of (2), it is well-known that $\varphi \in L^{\infty}\left(\boldsymbol{R}^{N}\right)$. Thus, by the definition of $\mathscr{L}$, we see that

$$
\left|\|\mathscr{L} \mathbf{v}\|_{L^{2}}-\|\Delta \mathbf{v}\|_{L^{2}}\right| \leq\|\mathscr{L} \mathbf{v}-i \Delta \mathbf{v}\|_{L^{2}} \leq C\|\mathbf{v}\|_{L^{2}}
$$

for all $\mathbf{v} \in H^{2}\left(\boldsymbol{R}^{N}, \boldsymbol{C}^{2}\right)$, where the constant $C$ depends on $\|\varphi\|_{L^{\infty}}$. This implies the desired inequalities.

Lemma 5. Let $N \leq 3$. There exists a constant $C$ such that

$$
\|F(\mathbf{v})\|_{L^{2}} \leq C\left(\|\mathbf{v}\|_{H^{2}}^{2}+\|\mathbf{v}\|_{H^{2}}^{p}\right)
$$

for all $\mathbf{v} \in H^{2}\left(\boldsymbol{R}^{N}, \boldsymbol{C}^{2}\right)$.

Proof. We put $g(v)=f(\varphi+v)-f(\varphi)-f^{\prime}(\varphi) v$. Then, by Lemma 3, we have

$$
\begin{aligned}
\|g(v)\|_{L^{2}} & =\left\|\int_{0}^{1}\left\{f^{\prime}(\varphi+\theta v)-f^{\prime}(\varphi)\right\} v d \theta\right\|_{L^{2}} \\
& \leq \int_{0}^{1}\left\|f^{\prime}(\varphi+\theta v)-f^{\prime}(\varphi)\right\|_{L^{\infty}}\|v\|_{L^{2}} d \theta \leq C\left(\|v\|_{H^{2}}^{2}+\|v\|_{H^{2}}^{p}\right) .
\end{aligned}
$$


Thus, we have

$$
\begin{aligned}
\|F(\mathbf{v})\|_{L^{2}} & \leq \gamma\left\|v_{1}\right\|_{L^{\infty}}\left(\left\|v_{1}\right\|_{L^{2}}+\left\|v_{2}\right\|_{L^{2}}\right)+\left\|v_{1}\right\|_{L^{\infty}}^{p-1}\left\|v_{1}\right\|_{L^{2}}+\left\|g\left(v_{2}\right)\right\|_{L^{2}} \\
& \leq C\left(\|\mathbf{v}\|_{H^{2}}^{2}+\|\mathbf{v}\|_{H^{2}}^{p}\right)
\end{aligned}
$$

for all $\mathbf{v} \in H^{2}\left(\boldsymbol{R}^{N}, \boldsymbol{C}^{2}\right)$.

Let $\gamma^{*}=\gamma^{*}(N, p, \omega)$ be the positive constant defined in Lemma 7 of [4]. The following lemmas are proved in [4].

Lemma 6 (Proposition 11 in [4]). Let $N \geq 1,1<p<1+4 / N$ and $\gamma>\gamma^{*}$. Then, there exist a constant $\lambda_{\gamma}>0$ and $\zeta_{\gamma} \in H^{2}\left(\boldsymbol{R}^{N}, \boldsymbol{C}\right)$ such that

$$
\left\|\zeta_{\gamma}\right\|_{H^{2}}=1, \quad \mathscr{L}_{1} \zeta_{\gamma}=\lambda_{\gamma} \zeta_{\gamma}, \quad \sup \left\{\Re \lambda: \lambda \in \sigma\left(\mathscr{L}_{1}\right)\right\}=\lambda_{\gamma} .
$$

Moreover, $\mathbf{z}_{\gamma}:=\left(\zeta_{\gamma}, 0\right)$ satisfies

$$
\mathscr{L} \mathbf{z}_{\gamma}=\lambda_{\gamma} \mathbf{z}_{\gamma}, \quad \sup \{\Re \lambda: \lambda \in \sigma(\mathscr{L})\}=\lambda_{\gamma} .
$$

Lemma 7 (Lemma 14 in [4]). Let $N \geq 1,1<p<1+4 / N$ and $\gamma>\gamma^{*}$. Let $\lambda_{\gamma}$ be the constant as in Lemma 6, and

$$
\alpha:=\frac{1}{2} \min \{1, p-1\}
$$

Then, there exists a constant $C$ such that

$$
\left\|e^{t \mathscr{L}} \mathbf{v}\right\|_{L^{2}} \leq C e^{(1+\alpha) \lambda_{y} t}\|\mathbf{v}\|_{L^{2}}
$$

for all $t \geq 0$ and $\mathbf{v} \in L^{2}\left(\boldsymbol{R}^{N}, C^{2}\right)$.

\section{Proof of Theorem 2}

For $\delta>0$, let $\vec{u}_{\delta}(t)$ be the solution of (1) with $\vec{u}_{\delta}(0)=(0,0, \varphi)+\delta\left(\zeta_{\gamma}, \zeta_{\gamma}, 0\right)$. Since $\varphi, \zeta_{\gamma} \in H^{2}\left(\boldsymbol{R}^{N}\right)$, we have $\vec{u}_{\delta}(0) \in H^{2}\left(\boldsymbol{R}^{N}, \boldsymbol{C}^{3}\right)$. Since $N \leq 3$ and $1<p<$ $1+4 / N$, we know that $\vec{u}_{\delta} \in C\left(\boldsymbol{R}, H^{1}\left(\boldsymbol{R}^{N}, \boldsymbol{C}^{3}\right)\right)$ (see Proposition 1 in [4]). Thus, by the $H^{2}$ regularity theorem for (1), we see that $\vec{u}_{\delta} \in C\left(\boldsymbol{R}, H^{2}\left(\boldsymbol{R}^{N}, \boldsymbol{C}^{3}\right)\right) \cap$ $C^{1}\left(\boldsymbol{R}, L^{2}\left(\boldsymbol{R}^{N}, \boldsymbol{C}^{3}\right)\right)$ (see, e.g., Section 5.3 in [1]). Moreover, by symmetry between $u_{1}$ and $u_{2}$ in (1), we see that $\vec{u}_{\delta}(t)$ has the form

$$
\vec{u}_{\delta}(t)=\left(e^{i \omega t} v_{\delta, 1}(t), e^{i \omega t} v_{\delta, 1}(t), e^{2 i \omega t}\left(\varphi+v_{\delta, 2}(t)\right)\right),
$$

where $\mathbf{v}_{\delta}(t):=\left(v_{\delta, 1}(t), v_{\delta, 2}(t)\right)$ is a solution of (5) with $\mathbf{v}_{\delta}(0)=\delta \mathbf{z}_{\gamma}$. Remark that $\mathbf{v}_{\delta} \in C\left(\boldsymbol{R}, H^{2}\left(\boldsymbol{R}^{N}, \boldsymbol{C}^{2}\right)\right) \cap C^{1}\left(\boldsymbol{R}, L^{2}\left(\boldsymbol{R}^{N}, C^{2}\right)\right)$, and $\mathbf{v}_{\delta}(t)$ satisfies the integral equation 


$$
\mathbf{v}_{\delta}(t)=\delta e^{\lambda_{\gamma} t} \mathbf{z}_{\gamma}+\int_{0}^{t} e^{(t-\tau) \mathscr{L}} F\left(\mathbf{v}_{\delta}(\tau)\right) d \tau
$$

for all $t \in \boldsymbol{R}$. Since

$$
\begin{aligned}
\partial_{t} \int_{0}^{t} e^{(t-\tau) \mathscr{L}} F\left(\mathbf{v}_{\delta}(\tau)\right) d \tau & =\partial_{t} \int_{0}^{t} e^{\tau \mathscr{L}} F\left(\mathbf{v}_{\delta}(t-\tau)\right) d \tau \\
& =e^{t \mathscr{L}} F\left(\mathbf{v}_{\delta}(0)\right)+\int_{0}^{t} e^{\tau \mathscr{L}} \partial_{t} F\left(\mathbf{v}_{\delta}(t-\tau)\right) d \tau \\
& =e^{t \mathscr{L}} F\left(\delta \mathbf{z}_{\gamma}\right)+\int_{0}^{t} e^{(t-\tau) \mathscr{L}} \partial_{\tau} F\left(\mathbf{v}_{\delta}(\tau)\right) d \tau
\end{aligned}
$$

we have

$$
\partial_{t} \mathbf{v}_{\delta}(t)=\lambda_{\gamma} \delta e^{\lambda_{\gamma} t} \mathbf{z}_{\gamma}+e^{t \mathscr{L}} F\left(\delta \mathbf{z}_{\gamma}\right)+\int_{0}^{t} e^{(t-\tau) \mathscr{L}} \partial_{\tau} F\left(\mathbf{v}_{\delta}(\tau)\right) d \tau .
$$

Lemma 8. There exists a constant $C$ such that

$$
\left\|\partial_{t} F\left(\mathbf{v}_{\delta}(t)\right)\right\|_{L^{2}} \leq C\left(\left\|\mathbf{v}_{\delta}(t)\right\|_{H^{2}}+\left\|\mathbf{v}_{\delta}(t)\right\|_{H^{2}}^{p-1}\right)\left\|\partial_{t} \mathbf{v}_{\delta}(t)\right\|_{L^{2}}
$$

for all $t \in \boldsymbol{R}$.

Proof. We put

$$
g(v)=f(\varphi+v)-f(\varphi)-f^{\prime}(\varphi) v \quad \text { and } \quad \mathbf{v}_{\delta}(t)=\left(v_{\delta, 1}(t), v_{\delta, 2}(t)\right) .
$$

Since $\varphi$ does not depend on $t$, we have

$$
\partial_{t} g\left(v_{\delta, 2}(t)\right)=\left\{f^{\prime}\left(\varphi+v_{\delta, 2}(t)\right)-f^{\prime}(\varphi)\right\} \partial_{t} v_{\delta, 2}(t) .
$$

Thus, by Lemma 3, we have

$$
\begin{aligned}
\left\|\partial_{t} g\left(v_{\delta, 2}(t)\right)\right\|_{L^{2}} & \leq\left\|f^{\prime}\left(\varphi+v_{\delta, 2}(t)\right)-f^{\prime}(\varphi)\right\|_{L^{\infty}}\left\|\partial_{t} v_{\delta, 2}(t)\right\|_{L^{2}} \\
& \leq C\left(\left\|v_{\delta, 2}(t)\right\|_{H^{2}}+\left\|v_{\delta, 2}(t)\right\|_{H^{2}}^{p-1}\right)\left\|\partial_{t} v_{\delta, 2}(t)\right\|_{L^{2}}
\end{aligned}
$$

Therefore, we have

$$
\begin{aligned}
\left\|\partial_{t} F_{2}\left(\mathbf{v}_{\delta}(t)\right)\right\|_{L^{2}} \leq & 2 \gamma\left\|v_{\delta, 1}(t)\right\|_{L^{\infty}}\left\|\partial_{t} v_{\delta, 1}(t)\right\|_{L^{2}} \\
& +C\left(\left\|v_{\delta, 2}(t)\right\|_{H^{2}}+\left\|v_{\delta, 2}(t)\right\|_{H^{2}}^{p-1}\right)\left\|\partial_{t} v_{\delta, 2}(t)\right\|_{L^{2}} \\
\leq & C\left(\left\|\mathbf{v}_{\delta}(t)\right\|_{H^{2}}+\left\|\mathbf{v}_{\delta}(t)\right\|_{H^{2}}^{p-1}\right)\left\|\partial_{t} \mathbf{v}_{\delta}(t)\right\|_{L^{2}} .
\end{aligned}
$$

The estimate for $\left\|\partial_{t} F_{1}\left(\mathbf{v}_{\delta}(t)\right)\right\|_{L^{2}}$ can be obtained in the same way. 
Lemma 9. There exist positive constants $C_{1}, \ldots, C_{5}$ such that

$$
\begin{aligned}
&\left\|\mathbf{v}_{\delta}(t)\right\|_{H^{2}} \leq\left\|\partial_{t} \mathbf{v}_{\delta}(t)\right\|_{L^{2}}+C_{1}\left\|\mathbf{v}_{\delta}(t)\right\|_{L^{2}}+C_{2}\left(\left\|\mathbf{v}_{\delta}(t)\right\|_{H^{2}}^{2}+\left\|\mathbf{v}_{\delta}(t)\right\|_{H^{2}}^{p}\right) \\
&\left\|\mathbf{v}_{\delta}(t)-\delta e^{\lambda_{\gamma} t} \mathbf{z}_{\gamma}\right\|_{L^{2}} \leq C_{3} \int_{0}^{t} e^{(1+\alpha) \lambda_{\gamma}(t-\tau)}\left(\left\|\mathbf{v}_{\delta}(\tau)\right\|_{H^{2}}^{2}+\left\|\mathbf{v}_{\delta}(\tau)\right\|_{H^{2}}^{p}\right) d \tau \\
&\left\|\partial_{t} \mathbf{v}_{\delta}(t)\right\|_{L^{2}} \leq \lambda_{\gamma} \delta e^{\lambda_{\gamma} t}+C_{4}\left(\delta^{2}+\delta^{p}\right) e^{(1+\alpha) \lambda_{\gamma} t} \\
& \quad+C_{5} \int_{0}^{t} e^{(1+\alpha) \lambda_{\gamma}(t-\tau)}\left(\left\|\mathbf{v}_{\delta}(\tau)\right\|_{H^{2}}+\left\|\mathbf{v}_{\delta}(\tau)\right\|_{H^{2}}^{p-1}\right)\left\|\partial_{t} \mathbf{v}_{\delta}(\tau)\right\|_{L^{2}} d \tau
\end{aligned}
$$

for all $t \geq 0$.

Proof. By Lemmas 4 and 5 and by (5), we have

$$
\begin{aligned}
\left\|\mathbf{v}_{\delta}(t)\right\|_{H^{2}} & \leq\left\|\mathscr{L}_{\mathbf{v}_{\delta}}(t)\right\|_{L^{2}}+C_{1}\left\|\mathbf{v}_{\delta}(t)\right\|_{L^{2}} \\
& \leq\left\|\partial_{t} \mathbf{v}_{\delta}(t)\right\|_{L^{2}}+\left\|F\left(\mathbf{v}_{\delta}(t)\right)\right\|_{L^{2}}+C_{1}\left\|\mathbf{v}_{\delta}(t)\right\|_{L^{2}} \\
& \leq\left\|\partial_{t} \mathbf{v}_{\delta}(t)\right\|_{L^{2}}+C_{1}\left\|\mathbf{v}_{\delta}(t)\right\|_{L^{2}}+C_{2}\left(\left\|\mathbf{v}_{\delta}(t)\right\|_{H^{2}}^{2}+\left\|\mathbf{v}_{\delta}(t)\right\|_{H^{2}}^{p}\right)
\end{aligned}
$$

for all $t \geq 0$. Next, by (6) and by Lemmas 7 and 5 , we have

$$
\begin{aligned}
\left\|\mathbf{v}_{\delta}(t)-\delta e^{\lambda_{\gamma} t} \mathbf{z}_{\gamma}\right\|_{L^{2}} & \leq C \int_{0}^{t} e^{(1+\alpha) \lambda_{\gamma}(t-\tau)}\left\|F\left(\mathbf{v}_{\delta}(\tau)\right)\right\|_{L^{2}} d \tau \\
& \leq C_{3} \int_{0}^{t} e^{(1+\alpha) \lambda_{\gamma}(t-\tau)}\left(\left\|\mathbf{v}_{\delta}(\tau)\right\|_{H^{2}}^{2}+\left\|\mathbf{v}_{\delta}(\tau)\right\|_{H^{2}}^{p}\right) d \tau
\end{aligned}
$$

for all $t \geq 0$. Finally, recall that $\left\|\mathbf{z}_{\gamma}\right\|_{H^{2}}=\left\|\zeta_{\gamma}\right\|_{H^{2}}=1$. By (7) and by Lemmas 5,7 and 8 , we have

$$
\begin{aligned}
\left\|\partial_{t} \mathbf{v}_{\delta}(t)\right\|_{L^{2}} \leq & \lambda_{\gamma} \delta e^{\lambda_{\gamma} t}\left\|\mathbf{z}_{\gamma}\right\|_{L^{2}}+C e^{(1+\alpha) \lambda_{\gamma} t}\left\|F\left(\delta \mathbf{z}_{\gamma}\right)\right\|_{L^{2}} \\
& +C \int_{0}^{t} e^{(1+\alpha) \lambda_{\gamma}(t-\tau)}\left\|\partial_{\tau} F\left(\mathbf{v}_{\delta}(\tau)\right)\right\|_{L^{2}} d \tau \\
\leq & \lambda_{\gamma} \delta e^{\lambda_{\gamma} t}+C_{4}\left(\delta^{2}+\delta^{p}\right) e^{(1+\alpha) \lambda_{\gamma} t} \\
& +C_{5} \int_{0}^{t} e^{(1+\alpha) \lambda_{\gamma}(t-\tau)}\left(\left\|\mathbf{v}_{\delta}(\tau)\right\|_{H^{2}}+\left\|\mathbf{v}_{\delta}(\tau)\right\|_{H^{2}}^{p-1}\right)\left\|\partial_{t} \mathbf{v}_{\delta}(\tau)\right\|_{L^{2}} d \tau
\end{aligned}
$$

for all $t \geq 0$. This completes the proof. 
Proof of Theorem 2. Let $C_{1}, \ldots, C_{5}$ be the positive constants given in Lemma 9, and put

$$
C_{0}=C_{1}+2 \lambda_{\gamma}+4 C_{4}+1
$$

Let $0<\varepsilon_{0}<1$ be a constant satisfying

$$
\begin{gathered}
2 C_{0} \varepsilon_{0}<1, \quad 8\left(C_{2}+\frac{C_{1} C_{3}+2 C_{5}}{\alpha \lambda_{\gamma}}\right)\left(2 C_{0} \varepsilon_{0}\right)^{2 \alpha}<1, \\
\frac{8 C_{0} C_{3}}{\alpha \lambda_{\gamma}}\left(2 C_{0} \varepsilon_{0}\right)^{2 \alpha}<\left\|\zeta_{\gamma}\right\|_{L^{2}} .
\end{gathered}
$$

For $\delta \in\left(0, \varepsilon_{0}\right)$, we define

$$
T_{\delta}=\frac{1}{\lambda_{\gamma}} \log \frac{\varepsilon_{0}}{\delta}, \quad V_{\delta}(t)=\left\|\mathbf{v}_{\delta}(t)\right\|_{H^{2}}+\left\|\partial_{t} \mathbf{v}_{\delta}(t)\right\|_{L^{2}}
$$

Remark that $\varepsilon_{0}=\delta e^{\lambda_{\gamma} T_{\delta}}$ and $1+2 \alpha=\min \{2, p\}$. First, we prove that

$$
V_{\delta}(t) \leq 2 C_{0} \delta e^{\lambda_{\gamma} t}
$$

for all $0 \leq t \leq T_{\delta}$. Let $\tilde{T}_{\delta}$ be the supremum of $T$ such that (10) holds for all $0 \leq t \leq T$. Suppose that $\tilde{T}_{\delta}<T_{\delta}$. Then, by (8), we have

$$
V_{\delta}(t) \leq 2 C_{0} \delta e^{\lambda_{\gamma} t} \leq 2 C_{0} \delta e^{\lambda_{\gamma} T_{\delta}}=2 C_{0} \varepsilon_{0}<1
$$

for all $0 \leq t \leq \tilde{T}_{\delta}$. Thus, by Lemma 9 and (11) and recalling $\left\|\mathbf{z}_{\gamma}\right\|_{L^{2}} \leq 1$, we have

$$
\begin{aligned}
V_{\delta}(t) \leq & 2\left\|\partial_{t} \mathbf{v}_{\delta}(t)\right\|_{L^{2}}+C_{1}\left\|\mathbf{v}_{\delta}(t)\right\|_{L^{2}}+2 C_{2} V_{\delta}(t)^{1+2 \alpha} \\
\leq & \left(C_{1}+2 \lambda_{\gamma}\right) \delta e^{\lambda_{\gamma} t}+4 C_{4} \delta^{1+2 \alpha} e^{(1+\alpha) \lambda_{\gamma} t}+2 C_{2} V_{\delta}(t)^{1+2 \alpha} \\
& +2\left(C_{1} C_{3}+2 C_{5}\right) \int_{0}^{t} e^{(1+\alpha) \lambda_{\gamma}(t-\tau)} V_{\delta}(\tau)^{1+2 \alpha} d \tau .
\end{aligned}
$$

Using (11), we have

$$
\begin{aligned}
\int_{0}^{t} e^{(1+\alpha) \lambda_{\gamma}(t-\tau)} V_{\delta}(\tau)^{1+2 \alpha} d \tau & \leq\left(2 C_{0} \delta\right)^{1+2 \alpha} \int_{0}^{t} e^{(1+\alpha) \lambda_{\gamma}(t-\tau)} e^{(1+2 \alpha) \lambda_{\gamma} \tau} d \tau \\
& =\left(2 C_{0} \delta\right)^{1+2 \alpha} \frac{e^{(1+2 \alpha) \lambda_{\gamma} t}}{\alpha \lambda_{\gamma}} \leq\left(2 C_{0} \varepsilon_{0}\right)^{2 \alpha} 2 C_{0} \delta \frac{e^{\lambda_{\gamma} t}}{\alpha \lambda_{\gamma}}
\end{aligned}
$$


Then, by (12) and (8) and by the fact that $V_{\delta}(t)^{1+2 \alpha} \leq\left(2 C_{0} \varepsilon_{0}\right)^{2 \alpha} 2 C_{0} \delta e^{\lambda_{\gamma} t}$, we obtain

$$
\begin{aligned}
V_{\delta}(t) & \leq\left(C_{1}+2 \lambda_{\gamma}+4 C_{4} \delta^{\alpha} \varepsilon_{0}^{\alpha}\right) \delta e^{\lambda_{\gamma} t}+4\left(C_{2}+\frac{C_{1} C_{3}+2 C_{5}}{\alpha \lambda_{\gamma}}\right)\left(2 C_{0} \varepsilon_{0}\right)^{2 \alpha} C_{0} \delta e^{\lambda_{\gamma} t} \\
& \leq \frac{3}{2} C_{0} \delta e^{\lambda_{\gamma} t}
\end{aligned}
$$

for all $0 \leq t \leq \tilde{T}_{\delta}$. This contradicts the definition of $\tilde{T}_{\delta}$. Thus, we have $T_{\delta} \leq \tilde{T}_{\delta}$, so $(10)$ holds for all $0 \leq t \leq T_{\delta}$. Next, by Lemma 9 and by (9) and (12), we have

$$
\begin{aligned}
\left\|\mathbf{v}_{\delta}(t)-\delta e^{\lambda_{\gamma} t} \mathbf{z}_{\gamma}\right\|_{L^{2}} & \leq 2 C_{3} \int_{0}^{t} e^{(1+\alpha) \lambda_{\gamma}(t-\tau)} V_{\delta}(\tau)^{1+2 \alpha} d \tau \\
& \leq \frac{4 C_{0} C_{3}}{\alpha \lambda_{\gamma}}\left(2 C_{0} \varepsilon_{0}\right)^{2 \alpha} \delta e^{\lambda_{\gamma} t} \leq \frac{\delta}{2} e^{\lambda_{\gamma} t}\left\|\zeta_{\gamma}\right\|_{L^{2}}
\end{aligned}
$$

for all $0 \leq t \leq T_{\delta}$. In particular, since $\delta e^{\lambda_{\gamma} T_{\delta}}=\varepsilon_{0}$ and $\mathbf{z}_{\gamma}=\left(\zeta_{\gamma}, 0\right)$, we have

$$
\begin{aligned}
\varepsilon_{0}\left\|\zeta_{\gamma}\right\|_{L^{2}}-\left\|v_{\delta, 1}\left(T_{\delta}\right)\right\|_{L^{2}} & \leq\left\|v_{\delta, 1}\left(T_{\delta}\right)-\varepsilon_{0} \zeta_{\gamma}\right\|_{L^{2}} \\
& \leq\left\|\mathbf{v}_{\delta}\left(T_{\delta}\right)-\varepsilon_{0} \mathbf{z}_{\gamma}\right\|_{L^{2}} \leq \frac{\varepsilon_{0}}{2}\left\|\zeta_{\gamma}\right\|_{L^{2}}
\end{aligned}
$$

Therefore, we have

$$
\inf _{\theta \in \boldsymbol{R}, y \in \boldsymbol{R}^{N}}\left\|\vec{u}_{\delta}\left(T_{\delta}\right)-\left(0,0, e^{i \theta} \varphi(\cdot+y)\right)\right\|_{H^{1}} \geq\left\|v_{\delta, 1}\left(T_{\delta}\right)\right\|_{L^{2}} \geq \frac{\varepsilon_{0}}{2}\left\|\zeta_{\gamma}\right\|_{L^{2}}
$$

Since $\left\|\vec{u}_{\delta}(0)-(0,0, \varphi)\right\|_{H^{1}}=\delta\left\|\left(\zeta_{\gamma}, \zeta_{\gamma}, 0\right)\right\|_{H^{1}} \rightarrow 0$ as $\delta \rightarrow 0$, this means that $\left(0,0, e^{2 i \omega t} \varphi\right)$ is orbitally unstable.

\section{References}

[1] Cazenave, T., Semilinear Schrödinger equations, Courant Lecture Notes in Mathematics, 10, American Mathematical Society, Providence, RI, 2003.

[2] Colin, M. and Colin, T., On a quasi-linear Zakharov system describing laser-plasma interactions, Differential Integral Equations, 17 (2004), 297-330.

[ 3 ] Colin, M. and Colin, T., A numerical model for the Raman amplification for laser-plasma interaction, J. Comput. Appl. Math., 193 (2006), 535-562.

[4] Colin, M., Colin, T. and Ohta, M., Stability of solitary waves for a system of nonlinear Schrödinger equations with three wave interaction, Ann. Inst. H. Poincaré Anal. Non Linéaire (in press).

[5] Ginibre, J. and Velo, G., Scattering theory in the energy space for a class of nonlinear wave equations, Comm. Math. Phys., 123 (1989), 535-573. 
[6] Grillakis, M., Shatah, J. and Strauss, W., Stability theory of solitary waves in the presence of symmetry, II, J. Funct. Anal., 94 (1990), 308-348.

[7] Kato, T., On nonlinear Schrödinger equations, Ann. Inst. H. Poincaré Phys. Théor., 46 (1987), 113-129.

[8] Tsutsumi, Y., Global strong solutions for nonlinear Schrödinger equations, Nonlinear Anal., 11 (1987), 1143-1154.

\author{
nuna adreso: \\ Mathieu Colin \\ Institut Mathématiques de Bordeaux \\ Université de Bordeaux \\ 351 cours de la libération \\ 33405 Talence Cedex \\ France \\ E-mail: mcolin@math.u-bordeaux1.fr \\ Thierry Colin \\ Institut Mathématiques de Bordeaux \\ Université de Bordeaux \\ 351 cours de la libération \\ 33405 Talence Cedex \\ France \\ E-mail: colin@math.u-bordeaux1.fr \\ Masahito Ohta \\ Department of Mathematics \\ Faculty of Science \\ Saitama University \\ Saitama 338-8570 \\ Japan \\ E-mail: mohta@mail.saitama-u.ac.jp
}

(Ricevita la 10-an de decembro, 2008) 